\title{
A comparison of the Wiltse versus midline approaches in degenerative conditions of the lumbar spine
}

\author{
John T. Street, MD, PhD, FRCSI, ${ }^{1}$ R. Andrew Glennie, MD, FRCSC, ${ }^{2}$ \\ Nicolas Dea, MSc, FRCSC, ${ }^{1}$ Christian DiPaola, MD, ${ }^{3}$ Zhi Wang, MD, MSc, FRCSC, ${ }^{4}$ \\ Michael Boyd, MD, MEng, FRCSC, ${ }^{1}$ Scott J. Paquette, MD, MEd, FRCSC, ${ }^{1}$ \\ Brian K. Kwon, MD, PhD, FRCSC, ${ }^{1}$ Marcel F. Dvorak, MD, FRCSC, ${ }^{1,4}$ and \\ Charles G. Fisher, MD, MHSc FRCSC ${ }^{1}$
}

\begin{abstract}
1Vancouver Spine Surgery Institute and Department of Orthopaedics, University of British Columbia, Vancouver, British Columbia; ${ }^{2}$ Division of Orthopedics, Dalhousie University, Halifax, Nova Scotia; ${ }^{4}$ Department of Surgery, University of Montreal, Montreal, Canada; and ${ }^{3}$ Department of Orthopedics, University of Massachusetts Medical Center, Worcester, Massachusetts
\end{abstract}

\begin{abstract}
OBJECTIVE The objective of this study was to determine if there is a significant difference in surgical site infection (SSI) when comparing the Wiltse and midline approaches for posterior instrumented interbody fusions of the lumbar spine and, secondarily, to evaluate if the reoperation rates and specific causes for reoperation were similar for both approaches.

METHODS A total of 358 patients who underwent 1- or 2-level posterior instrumented interbody fusions for degenerative lumbar spinal pathology through either a midline or Wiltse approach were prospectively followed between March 2005 and January 2011 at a single tertiary care facility. A retrospective analysis was performed primarily to evaluate the incidence of SSI and the incidence and causes for reoperation. Secondary outcome measures included intraoperative complications, blood loss, and length of stay. A matched analysis was performed using the Fisher's exact test and a logistic regression model. The matched analysis controlled for age, sex, comorbidities, number of index levels addressed surgically, number of levels fused, and the use of bone grafting.
\end{abstract}

RESULTS All patients returned for follow-up at 1 year, and adverse events were followed for 2 years. The rate of SSI was greater in the midline group ( 8 of 103 patients; 7.8\%) versus the Wiltse group (1 of 103 patients; $1.0 \%)(p=0.018)$. Fewer additional surgical procedures were performed in the Wiltse group ( $p=0.025 ; \mathrm{OR} 0.47 ; 95 \% \mathrm{Cl} 0.23-0.95)$. Proximal adjacent segment failure requiring reoperation occurred more frequently in the midline group (15 of 103 patients; $14.6 \%$ ) versus the Wiltse group ( 6 of 103 patients; $5.8 \%)(p=0.048$ ). Blood loss was significantly lower in the Wiltse group (436 ml) versus the midline group ( $703 \mathrm{ml})$; however, there was no significant difference between the 2 groups in intraoperative complications or length of stay.

CONCLUSIONS The patients who underwent the Wiltse approach had a decreased risk of wound breakdown and infection, less blood loss, and fewer reoperations than the midline patients. The risk of adjacent segment failure in short posterior constructs is lower with a Wiltse approach.

http://thejns.org/doi/abs/10.3171/2016.2.SPINE151018

KEY WORDS spine; Wiltse; midline; surgical approach; lumbar

$\mathrm{W}$ ITH an aging population and high expectations for a better quality of life, spinal fusion surgeries are being performed with increasing frequency in patients with significant medical and surgical comorbidities. ${ }^{11,12}$ Many of these patients have an increased risk of postoperative wound infection and reoperations and longer hospital stays with increased costs of care. , $^{1,20,23-25}$ As a result, surgeons are examining options to reduce surgical site infection, and in doing so have revisited the utility of the Wiltse paraspinal approach as a potential alternative to minimizing wound complications in this high-risk group of patients. ${ }^{2,3,6,8,11,16}$ 
The Wiltse approach to the posterior lumbar spine was first described in 1968. It was proposed as an alternate direct approach to the facet joint and transverse process, rather than the conventional midline approach..$^{34,35}$ As a muscle-splitting approach through the sacrospinalis muscle and between the multifidus and longissimus, the Wiltse approach theoretically leads to less tissue destruction and blood loss and potentially improved postoperative pain. With time, the approach evolved to be applicable to multiple indications with a growing number of proposed advantages, including the facilitation of 2 surgeons working concurrently, more direct pedicle screw insertion trajectories, easier decompression of far lateral disc herniations, and a surgical trajectory that enables decompression of the contralateral canal. ${ }^{7,36}$ One of the potential limitations of the Wiltse approach is that central decompression is technically more difficult to achieve, but this is overcome as one gains familiarity with the approach. ${ }^{30,31}$

There are numerous studies that have evaluated the effect of dissection time and extent of surgical retraction during midline spine surgery on local muscle function, vascularity, and viability. Studies in animals and humans have consistently shown that prolonged paraspinal muscle retraction predisposes patients to increased pain and a higher degree of tissue necrosis and puts them at a potentially greater risk of infection. ${ }^{2,9,10,18}$ Methods for minimizing soft-tissue injury have been proposed. Minimally invasive technologies have taken advantage of the paraspinal approach by way of percutaneous access points.

In this study, we compared the paraspinal Wiltse approach to the standard posterior midline approach in patients who underwent 1- or 2-level decompression with interbody fusion of the lumbar spine. The primary objective was to determine the difference in the rates of postoperative surgical site infection through a rigorous matched analysis. Secondary objectives included comparing the reoperation rates and evaluating the specific causes for reoperation.

\section{Methods \\ Study Design}

A retrospective cohort of consecutive patients with prospectively collected data who were admitted for surgical management of degenerative lumbar spine conditions were identified and enrolled between March 2005 and January 2011. Only patients with a full data complement over a 2-year follow-up period were included. Our methods for prospectively collecting adverse event data have been previously published. ${ }^{19,27,28}$ All patients underwent decompression and pedicle screw instrumentation through either a standard midline or Wiltse approach. The standard technique with either approach at our institution is to perform a complete facetectomy of at least 1 of the facets and insert the interbody cage through the foramen (transforaminal lumbar interbody fusion) while visualizing and protecting the exiting and traversing nerve roots. All patients had an interbody device inserted. Patients underwent a complete laminectomy if a midline approach was used, or a full laminectomy if a Wiltse approach was chosen. A standard Wiltse interval through the longissimus and multifidus was used, and the facet joint capsule was ex- posed. After pedicle screw instrumentation, the facet joint was removed/osteotomized. If further central decompression was required, the soft tissues were dissected off the lamina more medially to allow for bilateral laminectomy and flavectomy while carefully protecting the dura and displacing the cauda contents ventrally. A full central decompression was achieved with this approach.

Those patients included for analysis had a primary diagnosis of degenerative lumbar pathology with instability (degenerative/isthmic spondylolisthesis or anticipated intraoperative facet resection) and required a 1- or 2-level instrumented fusion. Patients were excluded if they had a traumatic spinal injury, tumor, or preoperative neurological deficit or required noninstrumented fusion or greater than 2-level fusion. A $2 \%$ chlorhexidine with isopropyl alcohol skin preparation was used on all patients preoperatively at 1 day before their operation and immediately preoperatively. Standard antibiotic prophylaxis was also used in all patients.

\section{Patient Outcomes}

Patient age, sex, prior spine surgery, and medical comorbidities were recorded preoperatively using a standardized format. Patients were categorized into 1 of 5 age groups for the purposes of this analysis (age ranges 17-44, $45-54,55-64,65-74$, and $\geq 75$ years). These categories facilitated comparisons between more comparable patients with roughly equal numbers in each group. The total operative time and intraoperative and postoperative blood loss were recorded by a blinded observer. The number of levels fused, bone graft type/bone graft substitute, and incidence of intraoperative adverse events (e.g., unintentional/ incidental durotomy, hardware malposition requiring revision) were recorded. Bone morphogenetic protein (BMP) (Medtronic) was used at the surgeons' discretion. ${ }^{13,33}$ The length of stay in the hospital was calculated for both groups based on the day of admission and discharge. Finally, the spine surgical invasiveness index (SSII) was calculated for both groups to ensure that any treatment heterogeneity based on the diagnosis was accounted for by comparing similar cases in terms of their risk of infection. SSII was reported to ensure that each group was comparable based on the complexity of the surgical case, and SSII represents the best predictor for risk of infection. ${ }^{4,22}$

All study patients were evaluated clinically and radiographically at 3,6 , and 12 months following surgery. All clinical encounters for 2 years were included in the data analysis of this study. Our referral/re-referral patterns and geographic demographics are such that all subsequent clinical encounters would have presented back to our quaternary care center. Surgical site infection and the need for a repeat surgical procedure were recorded throughout the study period. Infection was recorded using the definition of the Centers for Disease Control and Prevention..$^{14} \mathrm{~A}$ wound infection was documented by positive intraoperative cultures and demonstrated erythematous skin changes and purulent discharge. A wound complication included wound dehiscence without positive intraoperative cultures with minimal erythema or discharge. When repeat surgery was needed during the study period, fusion was assessed radiographically or intraoperatively by the direct 
observation of any fusion mass present and mechanical manipulation of the surgical levels. ${ }^{17}$

\section{Statistical Analysis}

The statistical analysis was performed using the SPSS statistical software package (version 22.0; SPSS). We performed a matched analysis since we expected to find differences in patient characteristics between approaches. To ensure a more robust comparison, we controlled for potential confounders and analyzed the data using the Fisher's exact test, odds ratios, and 1 -sided $\mathrm{p}$ values.

For the matched analysis, 1 midline patient was matched to 1 Wiltse patient based on sex, age group at admission (17-44, 45-54, 55-64, 65-74, or $\geq 75$ years), preoperative comorbidities (diabetes, other, or none), number of index operative levels $(1,2$, or 3$)$, number of instrumented vertebral levels (1 or 2), and bone graft used (autograft only, autograft and synthetic autograft [i.e., not BMP], or BMP/ $\mathrm{BMP}$ only/none).

For the secondary analysis, a logistic regression model comparing the odds of an additional admission requiring repeat surgery versus the approach (midline or Wiltse) was performed. This was also done to control for as many variables as possible in a matched fashion. Finally, a tertiary analysis evaluating the reasons for reoperation was performed.

\section{Results}

A total of 358 patients were included in the final analysis. No patients were lost to follow-up at 1 year, and the adverse event and reoperation data were collected for a total of 2 years after the index procedure. Two hundred fifty-five patients underwent a midline approach, and 103 patients underwent bilateral Wiltse approaches. The most common indications for surgery were isthmic spondylolisthesis and degenerative spondylolisthesis. Table 1 lists the complete diagnostic details and primary surgical indications for all midline and Wiltse patients. There was a statistically significant difference in patient age, with the Wiltse group being younger by approximately 5 years (Wiltse 54.6 years vs midline 59.6 years; $p=0.002$ ). Wiltse patients differed in terms of the number of levels addressed surgically, in addition to the number of instrumented levels, demonstrating a significantly higher number of 1-level surgeries. Only 1-level surgery was performed in 81 of 103 Wiltse patients (78.6\%), whereas midline surgeries tended to address more vertebral levels; only 154 of 255 (60.4\%)

TABLE 1. Distribution of various pathologies addressed by approach*

\begin{tabular}{lcc}
\hline \multicolumn{1}{c}{ Diagnosis } & $\begin{array}{c}\text { Midline } \\
(\mathrm{n}=255)\end{array}$ & $\begin{array}{c}\text { Wiltse } \\
(\mathrm{n}=103)\end{array}$ \\
\hline Isthmic spondylolisthesis & $55(21.6)$ & $54(52.4)$ \\
\hline Degenerative spondylolisthesis & $134(52.5)$ & $35(34.0)$ \\
\hline Spinal stenosis w/o spondylolisthesis & $40(15.7)$ & $5(4.9)$ \\
\hline Disc protrusions & $16(6.3)$ & $8(7.8)$ \\
\hline Other & $10(3.9)$ & $1(1.0)$ \\
\hline
\end{tabular}

* Values are presented as the number of patients (\%). patients underwent 1-level instrumented surgery as the index procedure $(\mathrm{p}<0.001)$.

BMP augmented with a local autograft was used significantly more often in the Wiltse group $(60.2 \%)$ than in the midline group $(43.9 \%)(\mathrm{p}<0.0001)$. Midline patients also relied on local autograft alone $(46.3 \%)$ for a greater majority of cases $(\mathrm{p}<0.0001)$. No other differences existed with regard to using BMP alone or another synthetic bone graft (Table 2). A mixture of BMP and local bone autograft was placed within the interbody device and along the transverse processes after decortication. There were no significant differences in the use of iliac crest bone graft between the groups $(p=0.865)$.

There was no difference in the mean length of the procedure, with midline surgeries lasting roughly 4.1 hours and Wiltse surgeries lasting 3.9 hours $(\mathrm{p}=0.263)$. Intraoperative blood loss was significantly lower in the Wiltse group (average $436 \mathrm{ml}$ ), whereas the midline group lost on average $703 \mathrm{ml}(\mathrm{p}<0.001)$. The incidental durotomy rate was the same for each group (4.5\% midline vs $4.9 \%$ Wiltse; $p=0.805)$. The average length of stay for midline patients was 1.5 days longer than the Wiltse group. Although this finding is clinically significant, it was not found to be statistically significant $(p=0.069)$. There was no significant difference in patients requiring a repeat surgery during their hospital stay for infection, hardware malposition, or pseudomeningocele $(p=0.363)$. Table 3 details all variables that were compared and demonstrates that there were no other significant differences when comparing approaches. The groups were similar in terms of complexity, and SSII was $10.74 \pm 2.63$ for the Wiltse group and $10.54 \pm 2.72$ for the midline group.

To additionally control for these differences, a matched analysis was then performed. It was possible to match $66 \%$ of the cohort for all 6 possible confounding variables, and $85.5 \%$ of the cohort was matched for 5 or more variables (sex, age, preoperative comorbidities, number of index operative vertebral levels, number of instrumented vertebral levels, and bone graft used). For example, $85.5 \%$ of patients were matched for sex, age, preoperative comorbidities, number of index operative vertebral levels, and number of instrumented vertebral levels, but $15 \%$ of patients could not be matched with respect to the type of bone graft used.

Case-control analysis of the matching cohorts revealed a lower infection rate in the Wiltse group (1 of 103 patients; $1.0 \%$ ) in comparison with the midline group ( 8 of 103 patients; 7.8\%) $(\mathrm{p}=0.018)$. The odds ratios for each of the control variables are shown in Table 4.

Patients who underwent the Wiltse approach demon-

TABLE 2. Distribution of patients who received bone graft or bone graft substitute to promote fusion*

\begin{tabular}{lcc}
\hline \multicolumn{1}{c}{ Bone Graft } & Midline & Wiltse \\
\hline Autograft only & $118(46.3)$ & $25(24.3) \dagger$ \\
\hline Autograft \& synthetic (no BMP) & $12(4.7)$ & $2(1.9)$ \\
\hline Autograft \& BMP & $112(43.9)$ & $62(60.2) \dagger$ \\
\hline BMP only & $12(4.7)$ & $13(12.6)$ \\
\hline
\end{tabular}

* Values are presented as the number of patients (\%).

$\dagger$ Significant at $p<0.0001$. 
TABLE 3. Detailed comparisons between each technique

\begin{tabular}{|c|c|c|c|c|}
\hline Variable & Wiltse & Midline & $\begin{array}{l}\text { atistical Test Comparing } \\
\text { Approaches }\end{array}$ & p Value \\
\hline Sex & & & Fisher's exact test & 0.292 \\
\hline Male & 53 & 114 & & \\
\hline Female & 50 & 153 & & \\
\hline Age at admission, yrs & 54.6 & 59.6 & Fisher's exact test & $0.002^{*}$ \\
\hline Previous spine surgery & & & Fisher's exact test & 1.000 \\
\hline Yes & 27 & 68 & & \\
\hline No & 76 & 187 & & \\
\hline No. of index procedure vertebral levels & & & Fisher's exact test & $<0.001^{*}$ \\
\hline 2 & 81 & 154 & & \\
\hline 3 & 21 & 101 & & \\
\hline 4 & 1 & 0 & & \\
\hline No. of index procedure instrumented vertebral levels & & & Fisher's exact test & $0.006^{*}$ \\
\hline 2 & 81 & 162 & & \\
\hline 3 & 22 & 93 & & \\
\hline Iliac crest bone graft used & & & Fisher's exact test & 0.865 \\
\hline Yes & 13 & 35 & & \\
\hline No & 90 & 220 & & \\
\hline$>1$ surgery required during stay & & & Fisher's exact test & 0.363 \\
\hline Yes & 2 & 22 & & \\
\hline No & 101 & 244 & & \\
\hline CSF leakage/dural tear & & & Fisher's exact test & 0.805 \\
\hline Yes & 5 & 16 & & \\
\hline No & 98 & 239 & & \\
\hline Additional admission requiring repeat surgery & & & Fisher's exact test & $0.006^{*}$ \\
\hline Yes & 12 & 63 & & \\
\hline No & 91 & 192 & & \\
\hline Intensive care unit stay & & & Fisher's exact test & 0.383 \\
\hline Yes & 6 & 9 & & \\
\hline No & 97 & 246 & & \\
\hline Recoded comorbidities (diabetes, other) & & & Fisher's exact test & 0.645 \\
\hline Yes & 32 & 79 & & \\
\hline No & 71 & 176 & & \\
\hline Distant-site infection & & & Fisher's exact test & 0.450 \\
\hline Urinary tract infection & 5 & 19 & & \\
\hline Bacteremia & 0 & 1 & & \\
\hline Pneumonia & 1 & 0 & & \\
\hline None & 97 & 234 & & \\
\hline Readmission w/ infection after distant-site infection & & & Fisher's exact test & 1.000 \\
\hline Yes & 1 & 2 & & \\
\hline No & 102 & 253 & & \\
\hline Length of stay (days) & 6.0 & 7.5 & 2-sample t-test & 0.069 \\
\hline Index procedure operative time (hrs) & 3.9 & 4.1 & 2-sample t-test & 0.263 \\
\hline Index procedure blood loss (ml) & 436 & 703 & 2-sample t-test & $<0.001^{*}$ \\
\hline
\end{tabular}

* Statistically significant at $p<0.05$.

strated reduced odds (OR 0.47; 95\% CI 0.23-0.95) of an additional admission requiring repeat surgery compared with patients who underwent surgery through the midline approach.

Adjacent-segment pathology differed with approach;
15 of $103(14.6 \%)$ midline patients required revision surgery within 2 years of the index procedure. This result was again statistically significant compared with the 6 of 103 (5.8\%) Wiltse patients who required a repeat operation for adjacent-level pathology $(\mathrm{p}=0.048)($ Table 5). 
TABLE 4. Odds ratios for the variables used in the matched analysis

\begin{tabular}{lc}
\hline \multicolumn{1}{c}{ Variable } & OR $(95 \% \mathrm{Cl})$ \\
\hline Wiltse vs midline & $0.47(0.23-0.95)$ \\
\hline Male vs female & $1.37(0.79-2.38)$ \\
\hline Age group at admission in yrs (reference: $\geq 75$ yrs) & \\
\hline $17-44$ & $0.35(0.12-1.02)$ \\
\hline $45-64$ & $0.37(0.13-1.05)$ \\
\hline $55-64$ & $0.46(0.18-1.17)$ \\
\hline $65-74$ & $0.68(0.28-1.64)$ \\
\hline Recoded comorbidity (reference: none) & \\
\hline Diabetes & $0.55(0.21-1.41)$ \\
\hline Other & $0.75(0.37-1.52)$ \\
\hline 2 index procedure vertebral levels vs 3 \& 4 levels & $0.45(0.10-2.08)$ \\
\hline 2 index procedure instrumented vertebral levels vs & $1.20(0.26-5.56)$ \\
3 levels & \\
\hline Recoded index procedure bone graft (reference: \\
BMP only) \\
\hline Autograft only \\
\hline Autograft \& synthetic (not BMP) \\
\hline Autograft \& BMP & $0.78(0.23-2.61)$ \\
\hline
\end{tabular}

\section{Discussion}

We present the largest study comparing the Wiltse approach to the more conventional midline approach for decompression and fusion of the degenerative lumbar spine. Matched analysis revealed significant differences in complications when comparing these approaches. We have identified several advantages of the Wiltse approach, including lower infection risk, shorter hospital stay, fewer readmissions requiring surgery, and a lower risk of reoperation, specifically for adjacent-segment pathology within 3 years of the index surgery.

The potential to reduce the infection rate from $7.8 \%$ to $1.0 \%$ is statistically and clinically significant and has tremendous implications for optimizing infection prevention for 1- and 2-level fusion surgeries of the degenerative lumbar spine. By controlling for sex, age, preoperative comorbidities, number of instrumented vertebral levels, and the use of bone grafting, there is additional robustness to these results. Additionally, the SSII values for each group were similar, indicating that the complexities of each case were essentially the same for each group. This is important information because it indicates that each group was comparable despite not being matched for diagnosis. These findings would support implementing the Wiltse approach for the treatment of 1- or 2-level degenerative spinal pathologies of the lumbar spine. Although the infection rate for the midline patients may appear high, it is consistent with the previously published infection rate at our center and other recent publications that evaluated degenerative lumbar spondylolisthesis. . $^{5,21}$

Many potential barriers exist in the adoption of this surgical approach. Often, surgeons who are trained using a midline approach assume that decompression of central stenosis cannot be as adequately or safely performed from lateral to medial. There is a presumed risk of incidental
TABLE 5. Causes for reoperation*

\begin{tabular}{lcc}
\hline \multirow{2}{*}{ Variable } & \multicolumn{2}{c}{ Matched } \\
\cline { 2 - 3 } & Wiltse & Midline \\
\hline No. of patients & 103 & 103 \\
\hline Wound complication & $0(0)$ & $4(3.9)$ \\
\hline Complication of instrumentation & $3(2.9)$ & $7(6.8)$ \\
\hline Pseudarthrosis & $2(1.9)$ & $0(0)$ \\
\hline Adjacent-level pathology & $6(5.8) \dagger$ & $13(12.6) \dagger$ \\
\hline
\end{tabular}

* Values are presented as the number of patients (\%).

$\dagger$ Indicates a statistically significant difference in adjacent-level pathology with each approach.

durotomy or unintended exiting nerve root injury due to poor visualization. The results of our study, however, demonstrate that there was no significant increase in incidental durotomy or iatrogenic nerve root injury rates when using the Wiltse approach.

Minimally invasive approaches to the spine take advantage of a muscular split for purposes of decompression and instrumentation. For similar reasons, mostly due to the lack of surgeon familiarity, these approaches have not gained widespread use, as expected, over the past decade when the technology was originally introduced. A significant learning curve has been demonstrated when learning a new surgical technique in a number of publications, especially those related to neurological injury. ${ }^{26,29,32}$ We would propose that the same learning curve would not necessarily exist with the adoption of the Wiltse approach for 2 reasons. First, the Wiltse approach allows excellent visualization of the lateral bony anatomy and safe landmarks are readily identified. Second, this study was performed over the years in which the majority of surgeons at our center were learning the Wiltse approach. Our results demonstrate no major differences in intraoperative complications, which also included the malposition of hardware or repeat operations for inadequate decompressions. Surgeons' experience ranged from 5 to 23 years in independent practice.

Approaching the spine through the midline often results in subperiosteal dissection and heavy retraction of the muscular tissue, not only to expose the transverse processes but also to gain an acceptable trajectory for pedicle screw instrumentation. During this central subperiosteal dissection, in order to gain adequate excursion of the tissue, the midline incision is often extended both superiorly and inferiorly with additional dissection. It is possible that with this additional dissection the supraspinous, interspinous, and adjacent facet capsules are being violated unintentionally. There are many other studies that demonstrate much greater injury to the surrounding tissues with a midline approach. ${ }^{6,15}$ This is possibly the start of the cascade that leads to a greater incidence of adjacent segment pathology with the midline approach, which our data support. Also, a more lateral pedicle screw entry point has a much lower incidence of violating the adjacent facet and capsule of the caudal level. This process is multifactorial, however, and therefore we cannot draw any firm conclusions. 


\section{Limitations}

The major limitation of this study is the absence of health-related quality-of-life data. Although we have demonstrated a difference in infection rates and need for repeat surgery, we do not have clinical data to suggest that this made any impact on the overall patient outcomes at 1 and 2 years. We can make conclusions that the morbidity associated with repeat operations would be significant, but we have not demonstrated this with our data. Early on in this trial, there was some uncertainty that adequate central decompression could be obtained from a paraspinal approach. The procedure was initially used more in L5-S1 isthmic spondylolisthesis. As surgeons became more familiar with the paraspinal approach, utilization increased and more degenerative spondylolisthesis patients with central stenosis were treated with a midline approach. Pathological variation within this spectrum of degenerative cases, however, should not influence our primary outcome of infection. Further studies comparing each surgical approach are required if firm conclusions are to be made about the actual clinical outcomes. This is especially true when analyzing the length of stay with each procedure. Despite a shorter mean length of stay of 1.5 days, this was not statistically significant and may represent the lack of power to truly determine a difference in this outcome. Also, the analysis of adjacent-segment changes did not account for the preoperative status of the adjacent disc, and therefore we cannot make definitive statements regarding the exact role of fusion versus natural history in our observations of adjacent segment failure.

In addition, a limitation of this study is inherent to its design, in that although the data were prospectively collected, the analysis is retrospective. This introduces a potential source of bias, and although the use of matched data decreases the likelihood of making inappropriate comparisons, we were unable to match all variables that could potentially confound our results. This included the collection of some potential confounders such as body mass index. Future prospective analyses with tighter control and strict inclusion/exclusion criteria could potentially improve the validity of the results.

\section{Conclusions}

When feasible, the Wiltse or paraspinal muscle-splitting approach should be preferred for patients undergoing decompression and fusion of the lumbar spine, in particular for individuals with an increased risk of infection. There is a higher probability of adjacent-segment pathology in patients undergoing 1- or 2-level lumbar fusions with the midline approach. There were no differences in the reoperation rates for pseudarthrosis or complications of instrumentation in our matched data set.

\section{Acknowledgments}

We acknowledge Juliet Batke and the staff of the Vancouver Spine Research Program for assistance with the data collection and analysis.

\section{References}

1. Abdul-Jabbar A, Takemoto S, Weber MH, Hu SS, Mum- maneni PV, Deviren V, et al: Surgical site infection in spinal surgery: description of surgical and patient-based risk factors for postoperative infection using administrative claims data. Spine (Phila Pa 1976) 37:1340-1345, 2012

2. Buttermann GR, Mullin WJ: Two-level circumferential lumbar fusion comparing midline and paraspinal posterior approach: 5-year interim outcomes of a randomized, blinded, prospective study. J Spinal Disord Tech 28:E534-E543, 2015

3. Cai FJ, Luo YC, Zhu JP, Yu XH, Liu XH, Li H, et al: [Surgical treatment of thoracolumbar burst fractures through Wiltse paraspinal approach.] Zhongguo Gu Shang 25:980-983, 2012 (Chinese)

4. Cizik AM, Lee MJ, Martin BI, Bransford RJ, Bellabarba C, Chapman JR, et al: Using the spine surgical invasiveness index to identify risk of surgical site infection: a multivariate analysis. J Bone Joint Surg Am 94:335-342, 2012

5. Dipaola CP, Saravanja DD, Boriani L, Zhang H, Boyd MC, Kwon BK, et al: Postoperative infection treatment score for the spine (PITSS): construction and validation of a predictive model to define need for single versus multiple irrigation and debridement for spinal surgical site infection. Spine J 12:218-230, 2012

6. Fan SW, Hu ZJ, Fang XQ, Zhao FD, Huang Y, Yu HJ: Comparison of paraspinal muscle injury in one-level lumbar posterior inter-body fusion: modified minimally invasive and traditional open approaches. Orthop Surg 2:194-200, 2010

7. Fraser RD, Hall DJ: Laminectomy combined with posterolateral stabilisation: a muscle-sparing approach to the lumbosacral spine. Eur Spine J 1:249-253, 1993

8. Fujibayashi S, Neo M, Takemoto M, Ota M, Nakamura T: Paraspinal-approach transforaminal lumbar interbody fusion for the treatment of lumbar foraminal stenosis. J Neurosurg Spine 13:500-508, 2010

9. Gejo R, Kawaguchi Y, Kondoh T, Tabuchi E, Matsui H, Torii $\mathrm{K}$, et al: Magnetic resonance imaging and histologic evidence of postoperative back muscle injury in rats. Spine (Phila Pa 1976) 25:941-946, 2000

10. German JW, Foley KT: Minimal access surgical techniques in the management of the painful lumbar motion segment. Spine (Phila Pa 1976) 30 (16 Suppl):S52-S59, 2005

11. Goz V, Weinreb JH, McCarthy I, Schwab F, Lafage V, Errico TJ: Perioperative complications and mortality after spinal fusions: analysis of trends and risk factors. Spine (Phila Pa 1976) 38:1970-1976, 2013

12. Gruskay JA, Fu M, Bohl DD, Webb ML, Grauer JN: Factors affecting length of stay following elective posterior lumbar spine surgery: a multivariate analysis. Spine J 15:1188-1195, 2015

13. Haft GF: Is off-label use of BMP in pediatric spine surgery now a standard of care? Commentary on an article by Amit Jain, MD, et al.: "Factors associated with use of bone morphogenetic protein during pediatric spinal fusion surgery. An analysis of 4817 patients." J Bone Joint Surg Am 95:e103,1e103.2, 2013

14. Horan TC, Gaynes RP, Martone WJ, Jarvis WR, Emori TG: CDC definitions of nosocomial surgical site infections, 1992: a modification of CDC definitions of surgical wound infections. Infect Control Hosp Epidemiol 13:606-608, 1992

15. Hyun SJ, Kim YB, Kim YS, Park SW, Nam TK, Hong HJ, et al: Postoperative changes in paraspinal muscle volume: comparison between paramedian interfascial and midline approaches for lumbar fusion. J Korean Med Sci 22:646-651, 2007

16. Jiang XZ, Tian W, Liu B, Li Q, Zhang GL, Hu L, et al: Comparison of a paraspinal approach with a percutaneous approach in the treatment of thoracolumbar burst fractures with posterior ligamentous complex injury: a prospective randomized controlled trial. J Int Med Res 40:1343-1356, 2012 
17. Kant AP, Daum WJ, Dean SM, Uchida T: Evaluation of lumbar spine fusion. Plain radiographs versus direct surgical exploration and observation. Spine (Phila Pa 1976) 20:2313-2317, 1995

18. Kawaguchi Y, Yabuki S, Styf J, Olmarker K, Rydevik B, Matsui H, et al: Back muscle injury after posterior lumbar spine surgery. Topographic evaluation of intramuscular pressure and blood flow in the porcine back muscle during surgery. Spine (Phila Pa 1976) 21:2683-2688, 1996

19. Kelly AM, Batke JN, Dea N, Hartig DP, Fisher CG, Street JT: Prospective analysis of adverse events in surgical treatment of degenerative spondylolisthesis. Spine J 14:2905-2910, 2014

20. Lee MJ, Cizik AM, Hamilton D, Chapman JR: Predicting medical complications after spine surgery: a validated model using a prospective surgical registry. Spine J 14:291-299, 2014, 2014

21. Liu J, Deng H, Long X, Chen X, Xu R, Liu Z: A comparative study of perioperative complications between transforaminal versus posterior lumbar interbody fusion in degenerative lumbar spondylolisthesis. Eur Spine J [epub ahead of print], 2015

22. Mirza SK, Deyo RA, Heagerty PJ, Konodi MA, Lee LA, Turner JA, et al: Development of an index to characterize the "invasiveness" of spine surgery: validation by comparison to blood loss and operative time. Spine (Phila Pa 1976) 33:2651-2662, 2008

23. Olsen MA, Nepple JJ, Riew KD, Lenke LG, Bridwell KH, Mayfield J, et al: Risk factors for surgical site infection following orthopaedic spinal operations. J Bone Joint Surg Am 90:62-69, 2008

24. Schairer WW, Carrer A, Deviren V, Hu SS, Takemoto S, Mummaneni P, et al: Hospital readmission after spine fusion for adult spinal deformity. Spine (Phila Pa 1976) 38:16811689,2013

25. Schoenfeld AJ, Carey PA, Cleveland AW III, Bader JO, Bono CM: Patient factors, comorbidities, and surgical characteristics that increase mortality and complication risk after spinal arthrodesis: a prognostic study based on 5,887 patients. Spine J 13:1171-1179, 2013

26. Sclafani JA, Kim CW: Complications associated with the initial learning curve of minimally invasive spine surgery: a systematic review. Clin Orthop Relat Res 472:1711-1717, 2014

27. Street JT, Lenehan BJ, DiPaola CP, Boyd MD, Kwon BK, Paquette SJ, et al: Morbidity and mortality of major adult spinal surgery. A prospective cohort analysis of 942 consecutive patients. Spine J 12:22-34, 2012

28. Street JT, Thorogood NP, Cheung A, Noonan VK, Chen J, Fisher CG, et al: Use of the Spine Adverse Events Severity System (SAVES) in patients with traumatic spinal cord injury. A comparison with institutional ICD-10 coding for the identification of acute care adverse events. Spinal Cord 51:472-476, 2013

29. Tian NF, Wu YS, Zhang XL, Xu HZ, Chi YL, Mao FM:
Minimally invasive versus open transforaminal lumbar interbody fusion: a meta-analysis based on the current evidence. Eur Spine J 22:1741-1749, 2013

30. Vialle R, Court C, Khouri N, Olivier E, Miladi L, Tassin JL, et al: Anatomical study of the paraspinal approach to the lumbar spine. Eur Spine J 14:366-371, 2005

31. Vialle R, Wicart P, Drain O, Dubousset J, Court C: The Wiltse paraspinal approach to the lumbar spine revisited: an anatomic study. Clin Orthop Relat Res 445:175-180, 2006

32. Villavicencio AT, Burneikiene S, Roeca CM, Nelson EL, Mason A: Minimally invasive versus open transforaminal lumbar interbody fusion. Surg Neurol Int 1:12, 2010

33. Walker B, Koerner J, Sankarayanaryanan S, Radcliff K: A consensus statement regarding the utilization of BMP in spine surgery. Curr Rev Musculoskelet Med 7:208-219, 2014

34. Watkins MB: Posterolateral bone-grafting for fusion of the lumbar and lumbosacral spine. J Bone Joint Surg Am 41:388-396, 1959

35. Wiltse LL, Bateman JG, Hutchinson RH, Nelson WE: The paraspinal sacrospinalis-splitting approach to the lumbar spine. J Bone Joint Surg Am 50:919-926, 1968

36. Wiltse LL, Spencer CW: New uses and refinements of the paraspinal approach to the lumbar spine. Spine (Phila Pa 1976) 13:696-706, 1988

\section{Disclosures}

The authors report the following. Dr. DiPaola owns stock in Nascent Surgical, works as a consultant for DePuy-Synthes Spine, receives support for non-study-related clinical or research efforts from Safe Passage Neuromonitoring, and has nonfinancial relationships with the New England Spine Study Group and Spinal Injury Research and Education Foundation. Dr. Fisher is a consultant for Medtronic and NuVasive and receives royalties through Medtronic. Dr. Street receives support for non-studyrelated clinical or research efforts through Medtronic and teaches for Medtronic.

\section{Author Contributions}

Conception and design: all authors. Acquisition of data: all authors. Analysis and interpretation of data: Street, Glennie, DiPaola, Wang, Boyd, Kwon, Dvorak, Fisher. Drafting the article: all authors. Critically revising the article: all authors. Reviewed submitted version of manuscript: all authors. Statistical analysis: Street, Glennie. Administrative/technical/material support: Street, Glennie, Dvorak, Fisher. Study supervision: Street, DiPaola, Dvorak, Fisher.

\section{Correspondence}

John Street, Vancouver Spine Surgery Institute, Blusson Spinal Cord Center, 818 West 10th Ave., Vancouver, BC V5Z1M9, Canada.email: john.street@vch.ca. 